\title{
Geometrical Pattern Recognition for Rearranging Chairs
}

\author{
Priyam Sharma, Shrikant Honade
}

\begin{abstract}
Currently, furniture is used by every person and all working people in their lives. In office hours, it is necessary for the people to keep the furniture organized after the completion of meeting or event. Now a day's every student, every employee and all the working people having rush in their life. So they forget to arrange their furniture in working space which will create bad look for professional environment and also affect the psychology. The common thoughts now a day in educational institutions is that after every laboratory session is over, the students left the chair unarranged and the corresponding lab assistant's need to arrange the chairs after session is over in order to keep laboratory ready for the upcoming sessions. For this situation, in this paper we proposed a robotic chair which move automatically and follow the tag. These tags are predefined and fixed at valve or table. Whenever program runs these chairs automatically goes to the tag nearby and will aligned at its original position.
\end{abstract}

Keywords: Object Tracking, Robotic Lab, Self-Positioning Chair, Image processing.

\section{INTRODUCTION}

Our objective is to arrange college lab chairs in a special way that will give a better look to the classrooms. Such participation will increase credibility of institute. Our main objective is to enhance the design of a professional laboratory. For this purpose, we have created a system based on image processing and a platform of Raspberry Pi. We use Raspberry Pi for the camera-interface which is very easy for us so we have easily interfaced the camera with hardware using the USB protocol we want to transfer [8]. We have many options like Omni wheels, Magnum wheels and Normal disc wheels for our system but we choose normal disc wheel for bulk order. We can create only the required system instead of the featured system. We had surveyed a fundamental problem in coaching institutes, corporate offices, every conference room or laboratory of various institutes in which we found that after every session no one went straight away [2]. On this, we surveyed this terrible problem, how institutions are facing such problems. The results of the survey surprised us a lot. Which was shared by us with lab assistants, support staff and administrative officials i.e. according to them after each 40 minutes of session he takes about 10 minutes to arrange his chairs for the next session?

Revised Manuscript Received on September 05, 2020.

* Correspondence Author

Priyam Sharma*, PG Student, Dept. of E \& TC, GHRU, Amravati, Maharashtra, Email: priyams654@gmail.com

Dr. Shrikant Honade, Assistant Professor, Dept. of E \& TC, GHRU, Amravati, Maharashtra, India. Email: shrikant.honade@raisoni.net

(c) The Authors. Published by Blue Eyes Intelligence Engineering and Sciences Publication (BEIESP). This is an open access article under the CC BY-NC-ND license (http://creativecommons.org/licenses/by-nc-nd/4.0/)
Earlier many models comes in market such as system which take reference from the ground so for a plane surface it will work properly[1], Ozkil system will work on desired station and whenever operator message him back even leg navigate [2]. Kamelia try to work dental chair automation which provide all the tools and required instruments nearby as per the requirement specific reason assistant from operation this is a good project but removal of human manpower will leads to advance technology but remove employment. To overcome such problems the robotic based wheel chair is designed as shown in figure 1 . In this experiment, we need to create an automated system to organize the records [5] in which the wheels and computers will create a system. The system will read its tag and once it is detected then the chair will start running for the intended position. We have started the work of creating an automatic chair under the creation of an automated-system for laboratories at colleges and institutional levels.

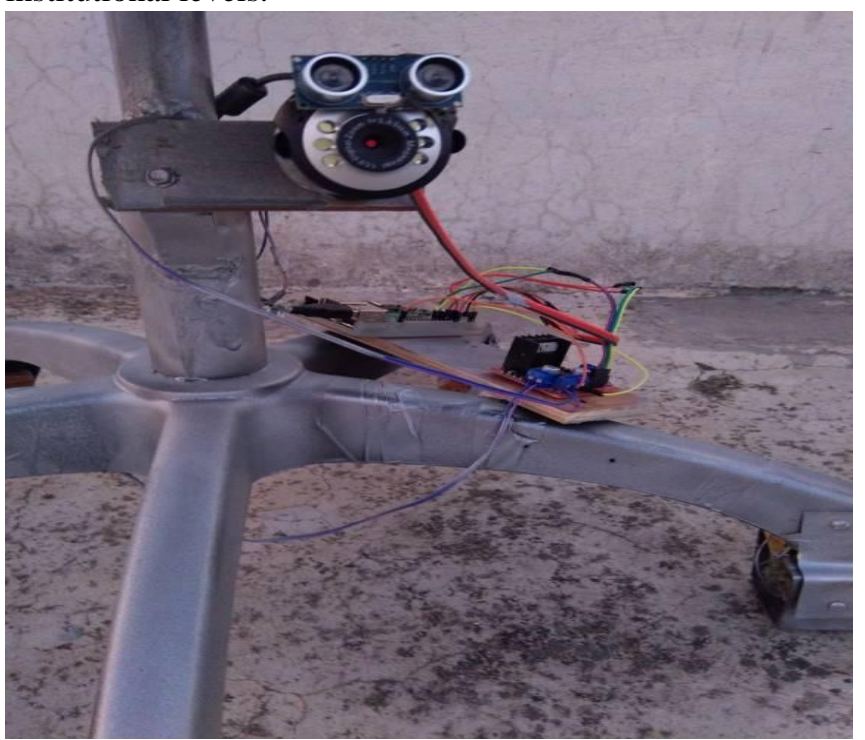

Figure 1: Designed model of a wheel chair setup

\section{METHOLOGY}

The methodology adopted for accomplishing the proposed work is mentioned below: Initially, we have made first virtual condition to distinguish explicit labels by the camera. Later we effectively introduced $\mathrm{CV}$ with testing of test labels handling. The apparatuses of the post-venture were utilized for picture preparing of Python's Open CV library. To start with, utilizing the Raspberry Pi Model 3B with 4GB SD card card storage [4]. So possibilities are very less that a man can wear pants with a letters in order print of any shading, yet it might be workable for more than one individual to wear similar shading

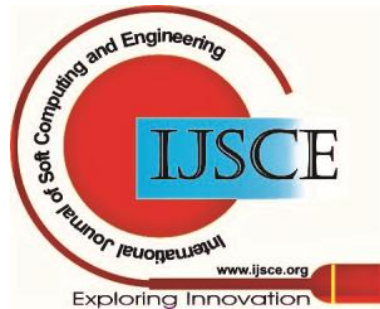




\section{Geometrical Pattern Recognition For Rearranging Chairs}

and structured dress simultaneously on any uncommon event, however normally incomprehensible. Exploiting this normal property, we will utilize ultrasonic sensors with Raspberry Pi, since ultrasound interfaces with ultrasonic sensors can be utilized at high recurrence by mirroring its wave from any item. This procedure will give a functioning favorable position over a slowed down seat, when any under study is situated, during this period which is viewed as sitting on the ultrasonic sensor since trigger heartbeat continuously got by Echo port inside separation of $20 \mathrm{~cm}$. These seats stay in the investigation position until nobody leaves seat.

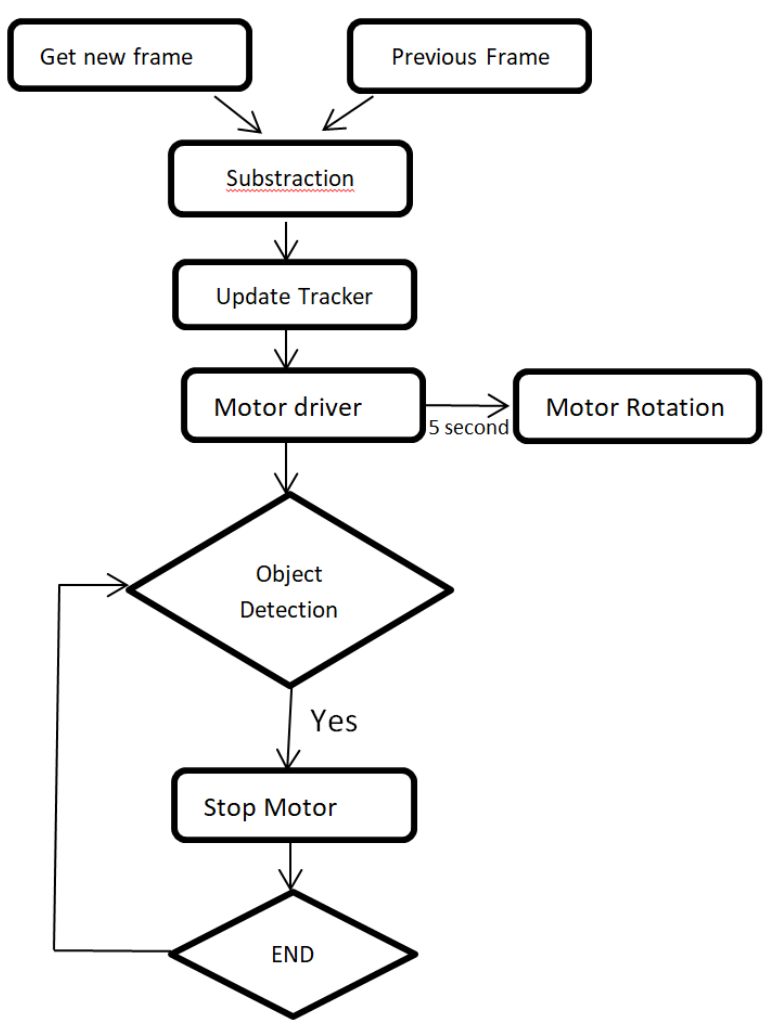

Figure 2: Flowchart of process

The detail procedure of working of the proposed system is depicted in figure 2. Here working of camera and ultra sonic sensor are extraordinary. Ultra sonic sensor just distinguish object comes in its range or not in extend. Engines work in combination with camera or sensor. State of ultra sonic sensor and ultrasonic casings are demonstrated to distinguish the size of the predefined tag after the individual is standing. The tag is set in the focal point of the edge and is $20 \mathrm{~cm}$ away from the first. The computerization framework has a different code set for the radius [11] which implies that the sensor's emphasis is on that code. On the off chance that the tag is to one side or right of the edge, the camera sees it and afterward moves the seat toward the tag and the seat starts to adjust toward the tag.

\section{II.1 Ramer Douglas Peucker algorithm:}

The Ramer-Douglas-Peucker algorithm, also known as the Douglas-Peucker algorithm and iterative end-point fit algorithm, is an algorithm that decimates a curve composed of line segments to a similar curve with fewer points. The starting curve is an ordered set of points or lines and the distance dimension $\varepsilon>0$ [8].

The algorithm recursively divides the line. Initially it is giving all the points between the first and last point. It automatically marks the first and last point to be kept. It then finds the point

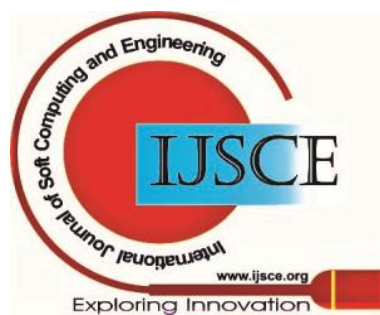




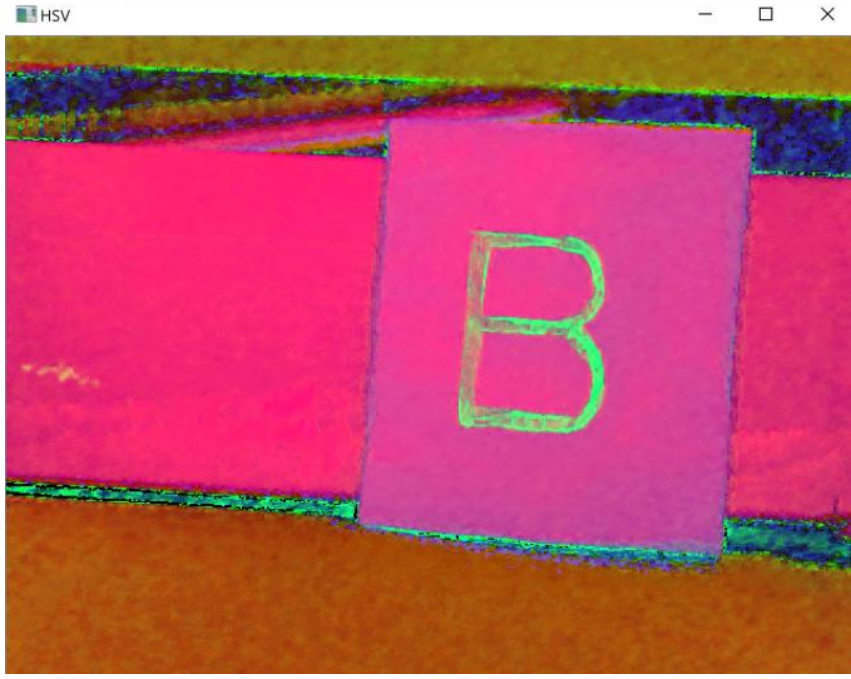

Figure 4: HSV Representation of LOGO

This procedure proceeded in light of the fact that once the framework is fueled on, it will keep on working in an unending circle of Python until we turn off the force. Be that as it may, for testing purposes we give a virtual showcase on the screen to distinguish the starting point and label conditions. We have the order office to choose this virtual showcase according to requirement [8]. These labels have an alternate character, distinctive size, and blend. These labels are structured explicitly for ventures that make exceptionally specific global positioning frameworks for each seat.

\section{RESULT \& DISCUSSION}

In this work, we have design and successfully tested the model by means of an RGB object tag tracking system using an open $\mathrm{CV}$ image processing tool for automated chairs and also performed a barrier detection test in front of the chair. The different tags position used during experimentation are shown in figure 5. So if any object discovered comes from the chair in front of the sensor system, the curse will stop and the chair will not move, because if someone is sitting on the chair then it will follow any tag to reduce the accident in the area at that time chair will not move.

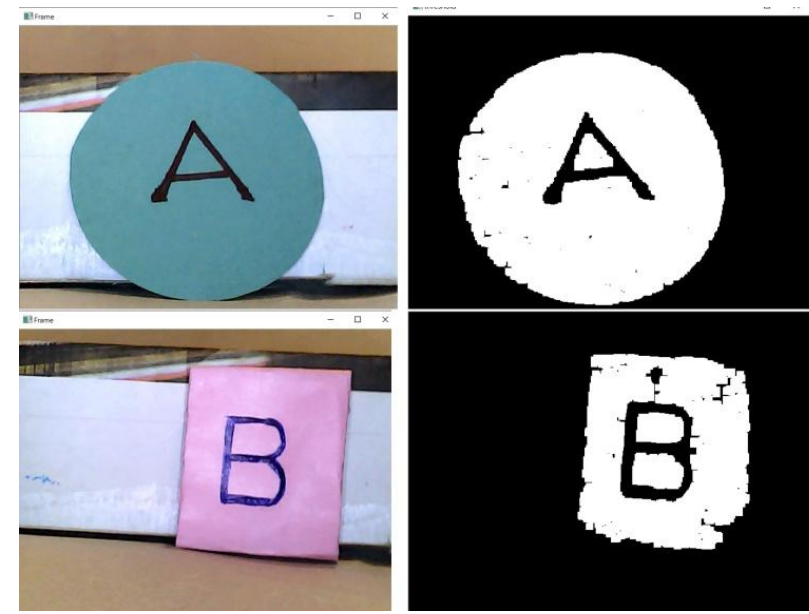

Figure 5: Position Tags

The reason behind is that the person closing the chair while using the curse and sometimes the color of the person's trousers is only green, blue, black, etc. which can cause an accident, as the system starts to follow the tag. But actually the tag is featured to prevent following the color of the trousers in the system which mainly the mixed use of Tag's shape, color and alphabet has been kept in the specific category, which will give very little chance that more than one person can hardly catch this mixture of Tag. The detail experimentation for the proposed system is accomplished by using different case studies related to the positional alignment of chair as under:

Case 1: When student simply left the chair by dragging it backward

In this case logo is centrally aligned with $Y$ axis. Here system assumes that in any point to $\mathrm{Y}$ axis logo can be situated among all the condition of logics. Then system finds logo position with respect to origin in its frame size. The figure 6 depicts the front position alignment.

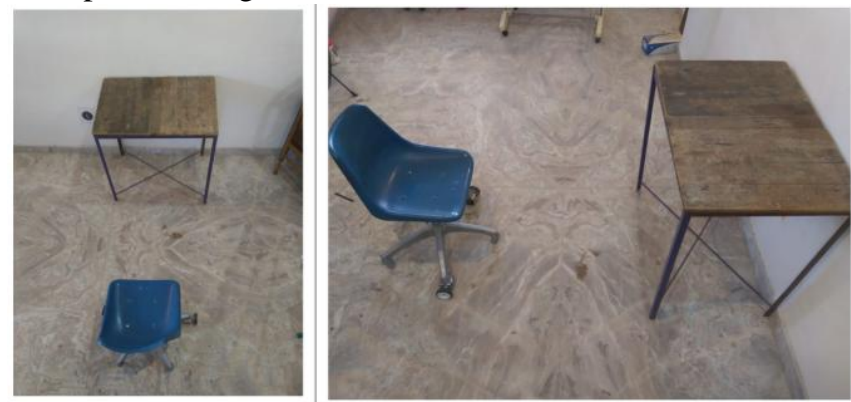

Figure 6: Front Position Alignment

In this case the system gives logical command which further converted into digital output using GPIO pins of Raspberry pi pins and send it to L293D motor driver IC. L293D takes external voltage from battery and passes it to motors with combination of HIGH \& LOW. Motors start moving after 3 second of count once the system starts. CPU continues adjusting logo position with respect to origin. Once logo is centrally aligned to chair then motors stops moving. That means chair is aligned at its intended position (under the table) and its stop moving.

Case 2: When student simply left the chair by dragging it Left / Right directed

When student left the chair by putting its position directed towards left or right side then in that case the chair starts moving and search for its tag to put it in frame. If it is not able to locate the tag then chair rotates round 360 degree container sleep for 10 seconds. The positional alignment for this case is depicted in figure 7.

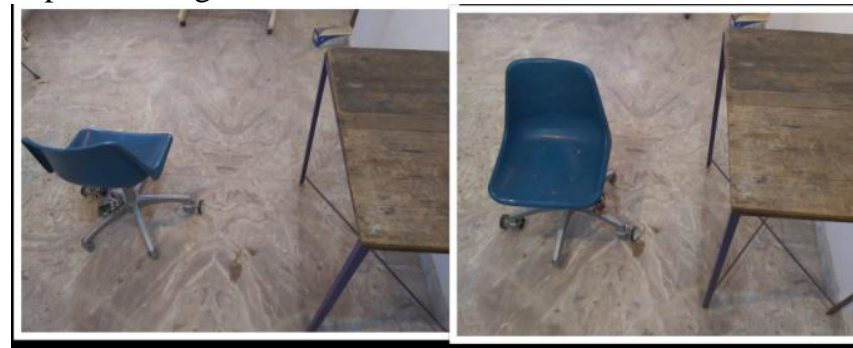

Figure 7: Front Position when tilted toward left/right

The benefit of this 360 degree turn is that during the term, the possibilities are increased off tag to come into frame position. So, system gives logical command which further converted into digital output using GPIO pins of Raspberry pi pins and send it to L293D.

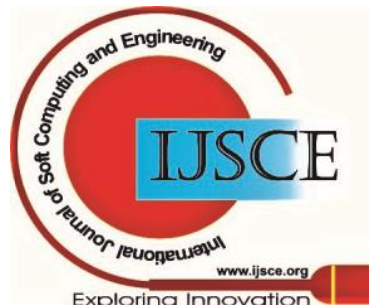




\section{Geometrical Pattern Recognition For Rearranging Chairs}

L293D take external voltage from battery and pass it to Motors with combination of HIGH \& LOW. Motors start moving after 3 second of count once system starts. CPU continues adjusting logo position with respect to origin. Once chair finds kept its logo in $\mathrm{Y}$ axis system start working according to case 1 .

\section{Case 3: When student comes between motions of chair}

When a student left his chair and chair start moving towards tag and in between if someone comes inside the range of chair then the ultrasonic sensor will gives trigger signal to raspberry pi and received signal will stop the chair. This process can prevent the proposed system for or regular accidents in laboratory. So, for this the system gives logical command which further converted into digital output using GPIO pins of Raspberry pi pins and send it to L293D. L293D take external voltage from battery and pass it to Motors with combination of LOW \& LOW. Motors stops working. Speed of processing is based on the $1 \mathrm{~GB}$ ram of raspberry pi therefor system is very fast. But due to moment of inertia motors will rotates $10 \%$ of its length.

\section{Case 4: When student simply left chair by dragging it opposite 360 degree}

When a student left his chair directed towards left or right side then chair starts moving and search for its tag to put in frame. If there is not able to locate the tag then chair rotates round 360 degree container sleep for 10 seconds. The diagrammatic representation for this case is shown in figure 8 .

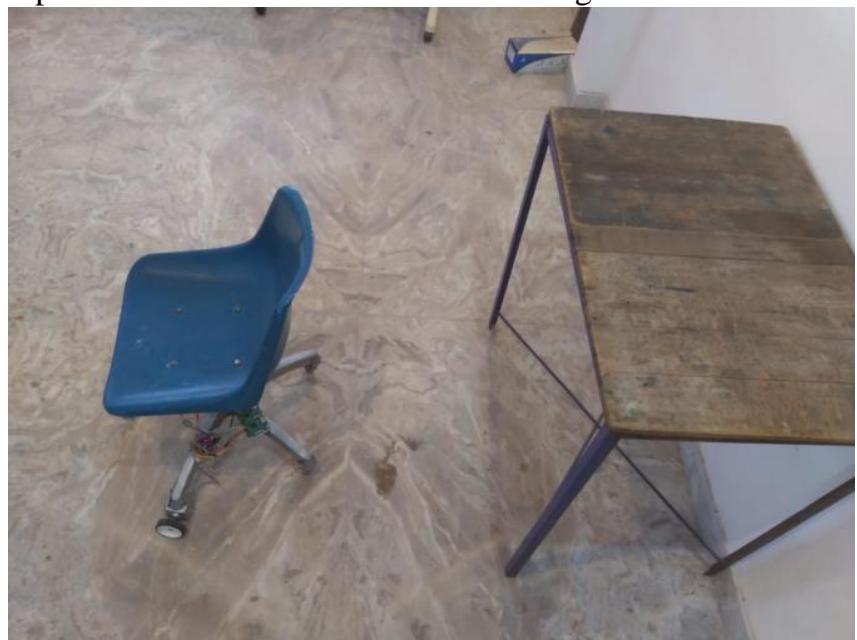

Figure 8: Extreme opposite Position

The benefit of this 360 degree turn is that during the term possibilities are increased off tag to come into frame position. So, system gives logical command which further converted into digital output using GPIO pins of Raspberry pi pins and send it to 1293d. L293D take external voltage from battery and pass it to Motors with combination of LOW \& HIGH. Motors start moving after 3 second of count once system starts. CPU continues adjusting logo position with respect to origin. Once chair finds kept its logo in $\mathrm{Y}$ axis system start working according to case 1 .

Our primary goal is to grow such a robotized framework which gives computerization in the undesirable human procedure. This model will help working environment composed and arranged style. When a gaggle of auto-labeling

recognition seats is organized by the robot in the areas of a research center or study hall, these can decrease the requirement for every day human endeavors in re-mastermind all the seats at their areas close to table. The proposed work is really finished for less expense as contrast with time estimation of individual worked in laboratory. This framework has capacity to choose its position utilizing self-labeling usefulness. At the point when the understudies leave the seats, these seats can work impeccably, subsequently increment the vibes of research facility. The presented prototype model is under implementation phase for practical laboratory at institute.

\section{REFERENCES}

1. B. Ross, "Office chair with automated height adjustment" Mar. 11 2008, uS Patent 7,341,310.

2. A. G. Ozkil "Design of a robotic automation system for transportation of goods in hospitals", IEEE, 2007, pp. 392-397.

3. L. Kamelia "Safety dental chair" N. R. Tatlock, uS Patent, 7,543,887.

4. R. Coleman, "Multi-feature automated wheelchair" Nov. 28 2000, uS Patent 6,154,690.

5. R. Freitas and P. Campos, "Smart: a system of augmented reality for teaching 2nd grade students," Proceedings of the 22nd British HCI Group Annual Conference on People and Computers: Culture, Creativity, Interaction, BCS Learning \& Development Ltd., Volume 2, 2008, pp. 27-30.

6. D. Kortenkamp, R. P. Bonasso, and R. Murphy “Artificial intelligence and mobile robots: case studies of successful robot systems". MIT Press, 1998.

7. M. Keertikumar, M. Shubham, and R. Banakar, "Evolution of iot in smart vehicles: An overview" in Green Computing and Internet of Things (ICGCIoT), 2015 International Conference on. IEEE, 2015, pp. 804-809.

8. T. Nam and T. A. Pardo, "Conceptualizing smart city with dimensions of technology, people, and institutions" in Proceedings of the 12th annual international digital government research conference: digital government innovation in challenging times. ACM, 2011, pp. 282291.

9. R. Kadam, P. Mahmauni, and Y. Parikh, "Smart home system" Interna- tional Journal of Innovative research in Advanced Engineering (IJIRAE), vol. 2, no. 1, 2015

10. T. Arsan, "Smart systems: from design to implementation of embedded smart systems," in HONET-ICT, 2016. IEEE, 2016, pp. 59-64.

11. G. Akhras, "Smart materials and smart systems for the future" Canadian Military Journal, vol. 1, no. 3, pp. 25- 31, 2000.

12. Agoston, Max K.. "Computer Graphics and Geometric Modeling: Implementation and Algorithms" Springer. pp. 300-306. ISBN 978-1-85233-818-3.

\section{BIOGRAPHIES}

\section{CONCLUSION}
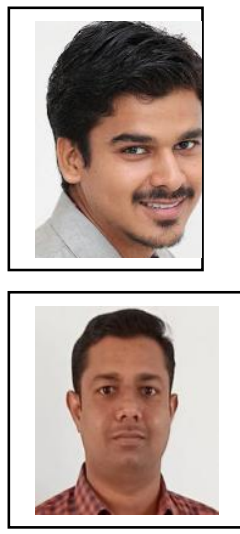

Mr. Priyam Sharma having Industrial experience of 5 years. Currently working as Director of DoxPro Robotics Pvt. Ltd. Also Pursing M. Tech. from GH Raisoni University. Amravati

Shrikant Honade has completed his Ph.D, M. Tech. and BE degree in Electronics \& Telecommunication from Sant Gadge Baba Amravati University Amravati, Maharashtra, India. He is presently working as a full time Assistant Professor in E \& TC department at G H Raisoni University, Amravati (M.S.), India. His research interest includes VLSI, DSP and AI.

Published By:

Blue Eyes Intelligence Engineering DOI:10.35940/ijsce.B3464.0910220

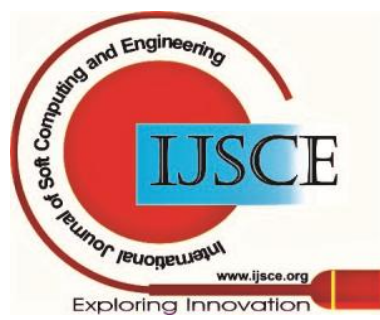

and Sciences Publication

(C) Copyright: All rights reserved. 